\title{
EXODONTIA SOB EFEITO HIPNÓTICO - RELATO DE CASO
}

\section{TOOTH EXTRACTION UNDER HYPNOTIC EFFECT - CASE REPORT}

\author{
landerlei Andrade Souza, Gabriel Bastos Teixeira, Juliano Fernandes Sassi, Gianny Roger \\ Parra Castanharo, Murilo Costas Rangel Pinheiro
}

Universidade Estadual do Sudoeste da Bahia - UESB

\begin{abstract}
Hypnotism has applications in different specialties such as Psychology, Dentistry, Surgery, Obstetrics, Oncology, chronic pain treatment, with different purposes and great results. The aim of this study was to use hypnosis as a complement to performan a dental extraction, seeking the control of anxiety and pain. Patient R.C.A.S., 50 years old, female, with extraction of the ' 36 ' element indication, under went seven sections of hypnosis until the extraction could be performed. After the procedure, the patient related that she did not have pain. The study showed that hypnosis can satisfactorily reduce anxiety and pain in cases of tooth extraction. More studies are needed to evaluate the effect of hypnosis in other oral and maxillofacial surgeries.
\end{abstract}

Keywords: Hypnosis. Hypnosis, Dental. Hypnosis, Anesthetic. Surgery, Oral

\section{Resumo}

O hipnotismo possui aplicações em diferentes especialidades como Psicologia, Odontologia, Cirurgia, Obstetrícia, Oncologia, tratamento da dor crônica, com diferentes finalidades e ótimos resultados. O objetivo desse trabalho foi utilizar a hipnose como complemento para realizar uma exodontia, buscando o controle da ansiedade $e$ da dor. Paciente R.C.A.S., 50 anos, sexo feminino, com indicação de extração da unidade 36, submeteu-se a sete seções de hipnose até poder realizar a extração. Concluído o procedimento, a paciente afirmou não ter sentindo dor. O estudo mostrou que a hipnose pode reduzir satisfatoriamente a ansiedade e a dor em casos de exodontia. Mais estudos são necessários para avaliar o efeito da hipnose em outras cirurgias bucomaxilofaciais.

Palavras-Chave: Hipnose. Hipnose em Odontologia. Hipnose Anestésica. Cirurgia Bucal 


\section{Introdução}

A hipnose possui uma vasta historiografia na medicina, psiquiatria e psicologia, mas não é tão extensa na odontologia. Isso é surpreendente, quando consideramos o importante papel que o hipnotismo teve na prática odontológica do século XIX e início do século $X X^{1}$. Considerado como o "Pai da Hipnose Científica", James Braid, no século XIX, obteve resultados terapêuticos por meio de métodos psicofisiológicos, batizando a técnica de "hipnose". Suas descobertas foram difundidas e permitiram o desenvolvimento de um método científico para o hipnotismo ${ }^{2}$. Entretanto, a hipnose é tão antiga quanto a origem da civilização e remonta aos tempos do Antigo Egito, onde os sacerdotes induziam sonos hipnóticos naqueles que buscavam alívio para os seus sofrimentos ${ }^{3}$. Outras civilizações antigas, como a hebraica e a celta, realizavam tratamentos com características muito semelhantes ${ }^{4}$.

Além de Braid, outras inúmeras personalidades científicas também aderiram à técnica e lutaram pelo seu reconhecimento, entre eles Mesmer, Puysegur, Faría, Esdaile, Bernheim, Charcort, Coué, Pávlov, Freud, Engels ${ }^{5,6,7}$.

O estado hipnótico pode dar-se de forma natural, de forma induzida por outra pessoa ou ser auto induzido e, por meio dele, o hipnotizador pode modular e controlar diversos processos, classificados como: virtuais, automáticos, viscerais e operacionais ${ }^{8,9}$.

Os processos virtuais são expressos na experiência subjetiva e consciente dos indivíduos, tais como a imaginação, situações, lembranças, percepções, delírios, alucinações, entre outros ${ }^{8,9}$. Já os processos automáticos são as condutas reflexas e os padrões emocionais ${ }^{8,9}$. Os processos viscerais são as dores, as sensações térmicas, a propriocepção, o equilíbrio, etc ${ }^{8,9}$. Por fim, os processos operacionais se dão através de respostas fisiológicas que envolvem o sistema motor do indivíduo, ou seja, sua coordenação motora $^{8,9}$.

Por esse motivo, o hipnotismo possui aplicações em diferentes especialidades como Psicologia, Odontologia, Cirurgia, Obstetrícia, Oncologia, tratamento da dor crônica, com diferentes finalidades e ótimos resultados ${ }^{10}$. Entretanto, a hipnose é uma área polêmica da investigação científica, uma vez que a experiência hipnótica ainda não recebeu explicações claras ${ }^{11}$.

Levando em consideração que o sonho de uma odontologia indolor tem sido profundamente enraizada no imaginário cultural dos pacientes por pelo menos dois séculos, e possui um grande significado pessoal e emocional para qualquer um que procura ou necessita de tratamento dentário ${ }^{1}$, utilizou-se a hipnose como um complemento para a cirurgia oral, uma vez que pode influenciar positivamente para bons resultados em pacientes com elevada ansiedade aos procedimentos odontológicos, em especial a exodontia.

O objetivo desse artigo consiste em relatar um caso de hipnose como complemento para a realização de exodontia e discutir a utilização e a eficácia da hipnose no controle da ansiedade e da dor durante tratamentos odontológicos, mais especificamente em casos de extração dentária.

\section{Metodologia}

Paciente R.C.A.S., 50 anos, sexo feminino, foi encaminhada ao consultório odontológico para extração da unidade 36. O exame radiográfico evidenciou pequena lesão radiolúcida de aspecto cístico no periápice do elemento, caracterizando uma ausência de vitalidade pulpar. A paciente relatou histórico de choque anafilático após o uso de anestesia geral e edema facial após uso de anestesia odontológica local. Devido às experiências traumáticas anteriores, a paciente revelou 0 desejo de ser submetida à hipnose para realizar a exodontia sem anestesia. Desse modo, a paciente passou a ser submetida a sessões semanais de hipnose para o controle da dor e ansiedade.

Nas duas primeiras sessões realizou-se um relaxamento corporal e introduziu-se a etapa hipnoidal, até a implantação do sinal hipnógeno. Já nas terceira e quarta sessões, iniciou-se a introdução do sinal hipnógeno e a paciente entrou imediatamente em transe hipnótico. A partir de então, realizou-se o aprofundamento da hipnose e a sugestão de anestesia.

Do mesmo modo, na quinta sessão, após estabelecimento do sinal hipnógeno, chegou-se a um maior aprofundamento da etapa sonambúlica, onde pôde-se observar fenômenos alucinatórios. Realizou-se outras duas sessões, chegando até a etapa de hipnose profunda.

O procedimento cirúrgico ocorreu após a paciente ser submetida ao sinal hipnógeno, chegando ao estado mais profundo, reforçada com estímulo hipnótico de anestesia e relaxamento muscular. Observada a ausência de sintomatologia dolorosa, utilizando teste de sensibilidade local, realizou-se a sindesmotomia no elemento 36 e iniciou-se o processo de luxação dentária com o fórceps. Após os 
primeiros movimentos, observou-se uma fratura coronária. Optou-se, então, pela odontosecção da unidade, utilizando-se broca multilaminada $\mathrm{n}$ ㅇ 703 em turbina de alta rotação, com irrigação copiosa. Seguiu-se a retirada das raízes utilizando-se alavancas apicais. Após a curetagem dos alvéolos para remoção da lesão cística e irrigação local com soro fisiológico a $0,9 \%$, realizou-se a sutura.

Concluído o procedimento e encerrada a hipnose, a paciente afirmou não ter ou estar sentindo dor.

\section{Resultados e Discussões}

Um estudo conduzido por Trakyali et al. ${ }^{12}$ avaliou a eficiência da hipnose consciente na colaboração de pacientes ortodônticos. Fizeram parte da pesquisa 30 indivíduos (14 mulheres e 16 homens) portadores de maloclusão Classe II Divisão 1 a e separados em dois grupos iguais, um grupo controle e um grupo de teste. Ambos os grupos receberam o mesmo tratamento ortodôntico, mas o grupo teste foi motivado com hipnose consciente e o grupo controle recebeu apenas motivação verbal do ortodontista. As análises estatísticas mostraram que a hipnose consciente é um método eficaz na melhora da cooperação do paciente ortodôntico. Até a data da realização desse estudo, ainda não haviam estudos que indicassem a relação entre o hipnotismo e a motivação dos pacientes odontológicos.

Em um relato de caso realizado por Ramazani, Zarenejad e Ebrahimi (2015) ${ }^{13}$, uma paciente do sexo feminino foi diagnosticada com esclerodermia no mesmo período em que realizava seu tratamento reabilitador oral, o que resultou na interrupção do mesmo devido a limitação de abertura de boca decorrente da doença autoimune. O impacto psicológico causado pela doença e a interrupção do tratamento odontológico acabaram causando um afastamento do convívio social. Desse modo, a paciente foi submetida a três sessões de hipnose para normalizar seu estado psicológico e facilitar a continuidade do tratamento odontológico. A partir do hipnotismo, a abertura de boca foi melhorada significativamente, e permitiu a conclusão do trabalho protético.

Uma pesquisa recente dirigida por Wannemueller et al. $(2011)^{14}$ comparou a eficácia e a aceitabilidade de quatro técnicas diferentes no tratamento de fobia dental - hipnose padronizada, hipnose com imagem individualizada, tratamento cognitivo comportamental e anestesia geral. Uma análise completa dos resultados mostrou uma significativa redução da ansiedade após o tratamento cognitivo comportamental e a hipnose individualizada comparados com o grupo de pacientes tratados com anestesia geral. No caso apresentado durante o condicionamento hipnótico para a extração foi feito a indução para redução da ansiedade, corroborando com o estudo acima.

Uma pesquisa desenvolvida por Abdeshahi et al. $(2013)^{15}$ avaliou a eficácia da hipnose no controle da ansiedade e da dor durante a extração de terceiros molares em 24 pacientes de ambos os sexos (14 homens e 10 mulheres), sem histórico médico significativo, onde o terceiro molar de um lado foi removido com a técnica anestésica local e o terceiro molar do lado oposto com a técnica hipnótica. No total, 48 molares foram extraídos e apenas dois pacientes submetidos à hipnose relataram dor depois da indução hipnótica, enquanto que oito pacientes submetidos à anestesia local relataram dor. Os resultados do estudo mostraram que os pacientes no grupo de hipnose tinham menos dor durante as primeiras horas de pósoperatório. Os escores de ansiedade nos dois grupos foram muito próximos uns dos outros e não houve diferença estatisticamente significante. No grupo de hipnose, dez pacientes $(41,7 \%)$ fizeram uso de medicação analgésica e no grupo da anestesia local, vinte e dois pacientes $(91,7 \%)$ tomaram a medicação, ou seja, comprovou-se que houve uma redução da dor, da hemorragia e da ansiedade com o uso do hipnotismo. Em consonância com estes resultados, no caso clinico apresentado a paciente relatou pouco incômodo pós operatório, nenhum sangramento e ansiedade.

Manzano et al. $(2013)^{16}$ realizou uma pesquisa para comprovar a eficiência do hipnotismo em casos de pacientes idosos com fobia dental. A amostra constituiu-se de vinte pacientes com sessenta anos ou mais, onde foram analisadas as variáveis de idade, sexo, número de sessões de hipnose, história médica, diagnósticos recentes e ansiedade. A hipnose ajudou a aliviar as tensões, o medo, a ansiedade na preparação pré-operatória, reduziu o estresse e evitou o aumento da pressão arterial durante todo o processo. O controle da ansiedade, medo e tensões foram analisados no caso apresentado.

O uso da hipnose na clínica diária tem suas limitações devido ao tempo clínico para se conseguir uma indução eficiente, mas em casos limites aonde se torna difícil o uso de anestésicos convencionais ou o paciente tem fobia à dentista 
acima do aceitável, a hipnose se torna uma alternativa de grande valia.

\section{Conclusões}

Os resultados do estudo mostraram que a hipnose pode reduzir satisfatoriamente ansiedade e a dor em casos de exodontia. Mais estudos são necessários para coletar dados sobre o efeito da hipnose em outros tipos de cirurgias bucomaxilofaciais.

\section{Referências}

1. Andrick JM. Cultivating a "chairsidemanner": dental hypnosis, patient management psychology, and the origins of behavioral dentistry in America, 1890-1910. Journal History Behavior Sci. 2013 Summer; 49(3):235-58.

2. Herreras E. Evaluación neuropsicológica en población adulta; instrumentos de evaluación. Cuaderno de Neuropsicología/Panamerican Journal of Neuropsychology. 2008; 2(2): 136-49.

3. Neubern M. Hipnose e psicologia clínica: retomando a história não contada. Psychology. 2006; 19(3): 346-54.

4. Williamson A. History of Hypnosis. In: Brann L, Owens J, Williamson A. The handbook of contemporary clinical hypnosis: theory and practice. London: Wiley-Blackwell; 2012.P. 31-40. 5. Martínez B, Asís M. Hipnosis. Teoría, métodos y técnicas. La Habana: Científico Técnica; 1995.

6. Freud S. Psicopatología de la vida cotidiana. Buenos Aires: Amorortu; 2001.

7. Marx K, Engels F. Obras escogidas. La Habana: Política; 1963.

8. Santibáñez-Hidalgo G, Osorio H. El objeto de la psicología II: los hechos psíquicos y el objeto de la psicología. Revista de Psicología. 1999; 8(1):89-104.

9. Santibáñez-hidalgo G. Funciones integrativas gnósticas del sistema neuroendocrino. Revista de Psicología. 2004; 13(2): 125-46.

10. Díaz J, Santibañez-Hidalgo G. Definiendo la hipnosis desde la psicobiología: algunas líneas de desarrollo científico de los fenómenos hipnóticos. Cuaderno de Neuropsicología/Panamerican Journal of Neuropsychology. 2008; 2(2):150-66.

11. Gonzáles J. Fisiología de lahipnosis. Cuadernos de Valoración. 2010; 10(1): 7-12.

12. Trakyali G, Sayinsu K, Müezzinoglu A, Arun T. Conscious hypnosis as a method for patient motivation in cervical headgear wear: a pilot study. Eur J Orthod. 2008; 30(1): 147-52.

13. Ramazani, M., Zarenejad, N., Ebrahimi, K. The help of hypnodontics to a patient with scleroderma. Iranian Journal of Psychiatry and Behavioral Sciences. 2015; 9(1): 1-3.

14. Wannemueller $A$, Joehren $P$, Haug $S$, Hatting M, Elsesser K, Sartory G. A practice-based comparison of brief cognitive behavioural treatment, two kinds of hypnosis and general anaesthesia in dental phobia. PsychotherPsychosom. 2011; 80(1): 159-65.

15. Abdeshahi $S$, Hashemipour $M$, Mesgarzadeh V, Payam A, Monfared A. Effect of hypnosis on induction of local anaesthesia, pain perception, control of haemorrhage and anxiety during extraction of third molars: a case-control study. J Craniomaxillofac Surg. 2013; 41(1): 31015.

16. Manzano E, Fernández A, Rodríguez A, Alvarado $M$, Dudefay $L$. Hipnosis como complemento en la cirugía oral del adulto mayor. Revista Cubana de Estomatología. 2013; 49(1): 41-52.

\section{Endereço para Correspondência}

Universidade Estadual de ponta Grossa - UEPG

Rua José Moreira Sobrinho - Jequiezinho, Jequié BA

CEP.: 45206-190

e-mail: ianderlei@ig.com.br

Recebido em 24/11/2017

Aprovado em 02/08/2018

Publicado em 12/09/2018 